\title{
Targeting TRAFs tames inflammation
}

Tumour necrosis factor receptorassociated factors (TRAFs) have a central role in inflammation and immunity. They function as adaptor proteins that link cell-surface receptors with downstream signalling cascades that ultimately result in the release of pro-inflammatory cytokines. Inhibition of TRAFs therefore represents a potential antiinflammatory therapeutic strategy. Now, writing in Nature Immunology, Mallampalli and colleagues report a novel role for F-box proteins in the regulation of TRAF levels and

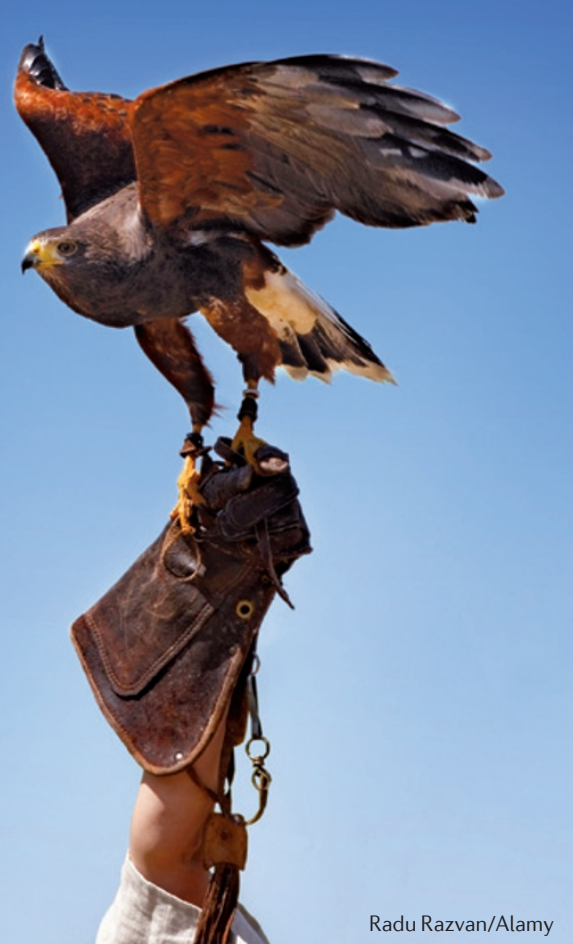

inflammation. They also identify a small-molecule inhibitor of FBXO3 that reduced cytokine-driven inflammation in several mouse models of infectious disease.

The F-box family of proteins are involved in various cellular functions, including protein ubiquitylation, where they form components of SCF ubiquitin ligase complexes.

FBXL2 is a recently identified member of the F-box family, and the authors found that FBXL2 expression in cells lowered levels of TRAFs, targeting these proteins for ubiquitylation and subsequent degradation. Further studies revealed that cells in which they overexpressed FBXL2 and exposed to inflammatory stimuli, such as lipopolysaccharide (LPS), displayed reduced TRAF protein levels and blunted cytokine release compared to control cells. Additional in vitro analysis showed that FBXL2 activity was regulated by another F-box protein, FBXO3, which triggers the ubiquitylation and degradation of FBXL2.

Analysis of a database of human single-nucleotide polymorphisms identified FBXO3(V221I) as a naturally occurring hypofunctional polymorphism. In human monocytes, the presence of this polymorphism engendered lower sensitivity to LPS stimulation and lower cytokine induction; this correlated with higher FBXL2 levels and lower TRAF levels. Interestingly, patients with sepsis had elevated levels of TRAFs, FBXO3 and pro-inflammatory cytokines and lower FBXL2 levels in the blood compared with control subjects and sepsis patients harbouring the FBXO3(V22I1) polymorphism.
Furthermore, when mice transfected with an Fbxo3(V221I)expressing plasmid or empty vector were challenged with Pseudomonas aeruginosa, they had reduced levels of cytokines and lung injury and higher survival rates compared with mice infected with a plasmid expressing wild-type Fbxo3. Similar anti-inflammatory effects were seen when $\mathrm{Fbxo3}$ was knocked down using short hairpin RNA.

Next, through structural analysis and molecular docking studies, the authors selected benzathine as a backbone for developing a potent small-molecule FBXO3 inhibitor: BC-1215. BC-1215 decreased the FBXO3-FBXL2 interaction in a dosedependent manner, prevented FBXL2 ubiquitylation and lowered TRAF levels in cells. Importantly, BC-1215 had an anti-inflammatory effect in several mouse models of infectious disease. BC-1215 significantly decreased circulating pro-inflammatory cytokine secretion in a sepsis model, it substantially ameliorated adverse effects of a $P$. aeruginosa infection on lung mechanics in a pneumonia model, and significantly reduced cell infiltrates and pro-inflammatory cytokine levels in an H1N1 pneumonia model.

Together, these studies reveal a novel role for F-box proteins in the regulation of inflammation and suggest FBXO3 as a target for a new anti-inflammatory therapeutic approach.

Sarah Crunkhorn

ORIGINAL RESEARCH PAPER Chen, B. B. et al. A combinatorial $\mathrm{F}$ box protein directed pathway controls TRAF adaptor stability to regulate inflammation. Nature Immunol. 14, 470-479 (2013) 